\title{
Succinate dehydrogenase expression in breast cancer
}

\author{
Sewha Kim, Do Hee Kim, Woo-Hee Jung and Ja Seung Koo*
}

\begin{abstract}
The aim of this study was to investigate succinate dehydrogenase (SDH) expression in breast cancer according to breast cancer molecular subtype using immunohistochemistry and to assess the clinical implications of SDH expression. Immunohistochemical staining for ER, PR, HER-2, Ki-67, HIF-1a, SDHA, and SDHB was performed on tissue microarrays of 721 breast cancers. According to the immunohistochemistry results for ER, PR, HER-2, and Ki-67 and fluorescence in situ hybridization (FISH) results for HER-2, breast cancer molecular subtypes were classified into luminal A, luminal B, HER-2, and triple-negative breast cancer (TNBC). HER-2 subtype breast cancers most frequently showed high-level expression of SDHA in tumor cells, while the luminal A subtype most frequently showed low or negative expression of SDHA in tumor cells $(P=0.032)$. Stromal SDHB expression rate was highest in HER-2 subtype and lowest in TNBC $(P<0.001)$. SDHA-negative breast cancers were associated with younger age at diagnosis $(P=0.012)$, and SDHB-negative breast cancers with lower histologic grade $(P=0.044)$ and lower Ki-67 labeling index $(\mathrm{LI})(P=0.046)$. Tumor phenotypes according to the $\mathrm{SDH}$ status were $\mathrm{SDHA}(+) / \mathrm{SDHB}(+)>\mathrm{SDHA}(-) / \mathrm{SDHB}$ $(-)>\mathrm{SDHA}(-) / \mathrm{SDHB}(+)>\mathrm{SDHA}(+) / \mathrm{SDHB}(-)$ in order of frequency. The stromal phenotypes were SDHA(-)/SDHB $(-)>\mathrm{SDHA}(+) / \mathrm{SDHB}(+)>\mathrm{SDHA}(-) / \mathrm{SDHB}(+)>\mathrm{SDHA}(+) / \mathrm{SDHB}(-)$. In conclusion, loss of SDHA or SDHB expression was observed in about 3\% of breast cancers in this study. Low SDH expression status in breast tumor cells was associated with younger age at diagnosis and low-grade histology.
\end{abstract}

Keywords: Breast; Metabolism; Mitochondria; Succinate dehydrogenase

\section{Introduction}

Succinate dehydrogenase (SDH) (succinate-coenzyme Q reductase, respiratory Complex II) catalyzes the oxidation of succinate to fumarate with the reduction of ubiquinone to ubiquinol. Through the coupling of these two reactions in the inner mitochondrial membrane, SDH links glucose oxidation in the TCA cycle with ATP production in the mitochondria (Cardaci \& Ciriolo 2012; King et al. 2006; Barletta \& Hornick 2012; Van Nederveen et al. 2009). SDH is composed of four subunits; SDHA, SDHB, SDHC and SDHD. The first two are hydrophilic proteins protruding into the mitochondrial matrix, and the second two are hydrophobic proteins that anchor the catalytic core into the inner mitochondrial membrane (Cardaci \& Ciriolo 2012; King et al. 2006; Barletta \& Hornick 2012; Van Nederveen et al. 2009).

\footnotetext{
* Correspondence: kjs1976@yuhs.ac

Department of Pathology, Yonsei University College of Medicine, Seoul, South Korea
}

Germline loss-of-function SDH mutations are associated with several tumors such as pheochromocytoma and paraganglioma, gastrointestinal stromal tumor (GIST), and renal cell carcinoma (Hensen \& Bayley 2011; Kantorovich et al. 2010; Anderson et al. 2004; Astuti et al. 2001; Miettinen et al. 2011; Badve et al. 2011; Ricketts et al. 2008). Among these, GIST and renal cell carcinoma are reported to display histologic features that distinguish SDH-deficient tumors (Miettinen et al. 2011). Although SDH germline mutation may be accurately evaluated by gene sequencing, a few recent studies have shown very high sensitivity for immunohistochemical detection of SDH mutation in GIST, pheochromocytoma, and paraganglioma (Van Nederveen et al. 2009; Gill et al. 2010a; Miettinen et al. 2013; Janeway et al. 2011).

To date, few studies have investigated SDH expression in breast cancer. We investigated SDH expression status according to breast cancer molecular subtype using immunohistochemical methods and assessed the clinical implications of SDH expression in breast cancer.

\section{首 Springer}




\section{Materials and methods Patient selection}

Patients who were diagnosed with invasive ductal carcinoma, not otherwise specified and underwent surgical excision at Severance Hospital between January 2002 and December 2005 were included in the study. Patients who received preoperative hormonal therapy or neoadjuvant chemotherapy were excluded. All hematoxylin and eosin (H\&E)-stained slides for each case were retrospectively reviewed by breast pathologists (Koo JS). The histological grade was assessed using the Nottingham grading system. (Elston \& Ellis 1991) The clinicopathologic parameters evaluated for each breast cancer included patient age at initial diagnosis, lymph node metastasis, tumor recurrence, distant metastasis, and patient survival. The Institutional Review Board of Yonsei University Severance Hospital approved this study.

\section{Tissue microarray}

On H\&E-stained slides of tumors, a representative area was selected and a corresponding spot was marked on the surface of the paraffin block. Using a biopsy needle, the selected area was punched out and a 3-mm core of tumor tissue was placed onto a $6 \times 5$ recipient block. Two tissue cores were extracted from each sample to minimize bias. Each tissue core was assigned a unique microarray location number that was linked to clinicopathologic data in a database.

\section{Immunohistochemistry}

All immunohistochemical staining was performed on formalin-fixed, paraffin-embedded tissue sections using antibodies as indicated (Table 1). Briefly, 5 - $\mu$ m-thick sections were obtained with a microtome, transferred onto adhesive slides, and dried at $62{ }^{\circ} \mathrm{C}$ for $30 \mathrm{~min}$. After incubation with primary antibodies, immunodetection was performed with biotinylated antimouse immunoglobulin, followed by peroxidase-labeled streptavidin using a labeled streptavidin biotin kit with 3,3'-diaminobenzidine chromogen as substrate. The primary antibody incubation step was omitted in the negative control. Slides were counterstained with Harris hematoxylin.

\section{Interpretation of immunohistochemical staining}

All immunohistochemical markers were assessed by light microscopy. Parameters such as ER, PR, and HER-2 status were obtained from patients' pathologic reports. A cut-off value of $1 \%$ or more positively stained nuclei was used to define ER and PR positivity (Hammond et al. 2010). HER-2 staining was analyzed according to the American Society of Clinical Oncology (ASCO)/College of American Pathologists (CAP) guidelines using the following categories: 0 , no immunostaining; $1+$, weak incomplete membranous staining, less than $10 \%$ of tumor
Table 1 Clone, dilution, and source of antibodies used

\begin{tabular}{llll}
\hline Antibody & Clone & Dilution & Company \\
\hline HIF-1a & EP1215Y & $1: 100$ & Biocare, Yorba Linda, CA, USA \\
SDHA & 2E3GC12FB2AE2 & $1: 100$ & Abcam, Cambridge, UK \\
SDHB & 21A11AE7 & $1: 100$ & Abcam, Cambridge, UK \\
Tumor phenotype-related & & \\
ER & SP1 & $1: 100$ & Thermo Scientific, CA, USA \\
PR & PgR & $1: 50$ & DAKO, Denmark \\
HER-2 & Polyclonal & $1: 1500$ & DAKO, Denmark \\
Ki-67 & MIB-1 & $1: 150$ & DAKO, Glostrup, Denmark \\
\hline
\end{tabular}

$E R$ estrogen receptor; $P R$ progesterone receptor, $E G F R$ epidermal growth factor receptor.

cells; $2+$, complete membranous staining, either uniform or weak in at least $10 \%$ of tumor cells; and $3+$, uniform intense membranous staining in at least $30 \%$ of tumor cells (Wolff AC, et al. 2007). HER-2 immunostaining was recorded as positive when strong $(3+)$ membranous staining was observed, whereas staining categories 0 to $1+$ were recorded as negative. Samples showing $2+$ HER-2 expression were evaluated for HER-2 amplification by fluorescence in situ hybridization (FISH).

Immunohistochemical staining for SDHA and SDHB showed cytoplasmic expression with a granular pattern. Complete loss of expression was considered negative, while cytoplasmic expression in less than $50 \%$ of tumor cells was considered low expression, and cytoplasmic expression in more than $50 \%$ of tumor cells was considered high expression (Figure 1). HIF-1 $\alpha$ was considered positive when more than $10 \%$ of tumor cells showed strong cytoplasmic or nuclear expression.

\section{Fish analysis}

FISH was performed using a PathVysion HER-2 DNA Probe Kit (Vysis, Downers Grove, IL, USA) according to the manufacturer's instructions. Invasive tumors were first examined on hematoxylin-eosin-stained slides to confirm histology. HER-2 gene copy number on the slides was evaluated using an epifluorescence microscope (Olympus, Tokyo, Japan) according to the ASCO/CAP guidelines (Wolff AC, et al. 2007). At least 60 tumor cell nuclei in three separate regions were investigated for HER-2 and chromosome 17 signals. An absolute HER-2 gene copy number lower than 4 or an HER-2 gene/chromosome 17 copy number ratio (HER-2/Chr17 ratio) less than 1.8 was considered HER-2-negative. An absolute HER-2 copy number between 4 and 6 or an HER-2/Chr17 ratio between 1.8 and 2.2 was considered HER-2-equivocal. An absolute HER-2 copy number greater than 6 or an HER-2/Chr17 ratio higher than 2.2 was considered HER-2positive. 


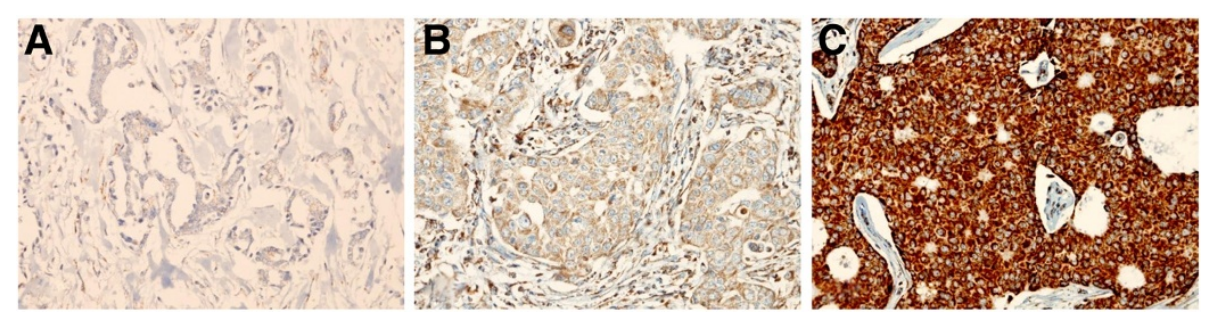

Figure 1 Immunohistochemical evaluation of SDHB expression. (A) negative (B) low positive (C) high positive.

\section{Tumor phenotype classification}

Breast cancer phenotypes were classified according to the immunohistochemistry results for ER, PR, HER-2, and Ki-67 and the FISH results for HER-2 as follows (Goldhirsch A, et al. 2011): Luminal A subtype as ERor/and PR-positive and HER-2-negative and Ki-67 LI $<14 \%$; Luminal B subtype as ER- or/and PR-positive and HER-2-negative and Ki-67 LI $\geq 14 \%$; HER-2 positive as ER- or/and PR-positive and HER-2 overexpressed or/ and amplified; HER-2-overexpression subtype as ERand PR-negative and HER-2 overexpressed or/and amplified; and triple-negative breast cancer (TNBC) subtype as ER-, PR-, and HER-2-negative.

\section{Statistical analysis}

Data were analyzed using SPSS for Windows, Version 12.0 (SPSS Inc., Chicago, IL, USA). Student's t and Fisher's exact tests were used to evaluate continuous and categorical variables, respectively. The significance level was set at 0.05 . The time to tumor recurrence and overall survival were evaluated by Kaplan-Meier and log-rank statistics. Multivariate regression analysis was performed using the Cox proportional hazards model.

\section{Results}

\section{Patients' characteristics}

Clinicopathological characteristics according to breast cancer molecular subtype are shown in Table 2. Molecular subtypes among these 721 breast cancers included 302 luminal A (41.9\%), 168 luminal B (23.3\%), 69 HER-2 type (9.6\%), and 182 TNBC (25.2\%). TNBC showed higher histologic grade $(P<0.001)$, higher T stage $(P=0.007)$, and higher Ki-67 LI $(P<0.001)$ than other molecular subtypes. The HER-2 subtype was positively associated with age $(P=0.005)$ and with tumor recurrence and death $(P=0.001)$.

HIF-1a and sdh status according to molecular subtype of breast cancer

HIF-1 $\alpha$, SDHA, and SDHB expression according to breast cancer subtype are shown in Table 3 and Figure 2. Only $24(3.3 \%)$ and $4(0.6 \%)$ of 721 breast cancers displayed HIF- $1 \alpha$ expression in the nucleus and cytoplasm, respectively. The HER-2 tumor subtype showed the highest frequency of high SDHA expression, and the luminal A subtype most frequently showed low or negative SDHA expression $(P=0.032)$. Stromal SDHB expression frequency was highest in the HER2 subtype and lowest in TNBC $(P<0.001)$. Stromal SDHA expression was detected most frequently in the HER-2 subtype, although the correlation was not significant $(P=0.063)$. Nuclear HIF- $1 \alpha$ expression and SDHA/SDHB expression did not correlate significantly (Table 4); however, SDHA and SDHB expression were correlated significantly $(r=0.895$, $P<0.001$, Figure 3 ).

\section{Clinicopathologic characteristics of breast cancer with SDHA and/or SDHB negativity}

Clinicopathologic characteristics of breast cancers were compared between the SDHA/SDHB positive and negative breast cancers (Table 5). Tumor negativity for SDHA correlated with earlier age at diagnosis of breast cancer $(P=0.012)$ and with lower histologic grade $(P=0.062)$. SDHB-negative breast cancer correlated with lower histologic grade $(P=0.044)$ and lower Ki-67 LI $(P=0.046)$. There was no correlation between the expression status of SDH and that of nuclear HIF- $1 \alpha$ (Table 4).

\section{Correlations between clinicopathologic parameters and expression of Hif- $1 \mathrm{a}$ and SDH}

Correlation analysis between HIF-1 $\alpha$ and SDH expression and clinicopathologic factors revealed an association of nuclear HIF- $1 \alpha$ expression with PR negativity $(P=0.011)$, and of low or negative SDHA expression in the tumor with ER positivity $(P=0.044)$, HER-2 negativity $(P=0.021)$, and higher $\mathrm{T}$ stage $(P=0.031)$. Low or negative SDHB expression in the tumor was associated with higher $\mathrm{T}$ stage $(P=0.011)$. Stromal SDHB negativity was associated with HER-2 negativity $(P<0.001$, Table 6$)$.

\section{Clnicopathologic features according the phenotype of $\mathrm{SDH}$ expression in breast cancer}

The SDHA/SDHB expression phenotypes in these 712 breast tumors were, in order of frequency, SDHA $(+) / \mathrm{SDHB}(+)>\operatorname{SDHA}(-) / \mathrm{SDHB}(-)>\operatorname{SDHA}(-) / \mathrm{SDHB}(+)>$ SDHA $(+) / \operatorname{SDHB}(-)$. The stroma phenotypes were SDHA 
Table 2 Clinicopathologic characteristics according to breast cancer phenotype

\begin{tabular}{|c|c|c|c|c|c|c|}
\hline Parameters & Total $n=721(\%)$ & $\begin{array}{c}\text { Luminal A } \\
n=302(\%)\end{array}$ & $\begin{array}{c}\text { Luminal B } \\
n=168(\%)\end{array}$ & $\begin{array}{c}\text { HER-2 } \\
n=69(\%)\end{array}$ & $\begin{array}{c}\text { TNBC } \\
n=182(\%)\end{array}$ & $P$-value \\
\hline Age (years, mean $\pm S D$ ) & $49.6 \pm 10.9$ & $50.6 \pm 10.4$ & $48.4 \pm 10.0$ & $52.4 \pm 10.0$ & $48.0 \pm 12.4$ & 0.005 \\
\hline Histologic grade & & & & & & $<0.001$ \\
\hline । & $120(16.6)$ & $92(30.5)$ & $20(11.9)$ & $1(1.4)$ & $7(3.8)$ & \\
\hline$\|$ & $364(50.5)$ & $182(60.3)$ & $91(54.2)$ & $36(52.2)$ & $55(30.2)$ & \\
\hline III & $237(32.9)$ & $28(9.3)$ & $57(33.9)$ & $32(46.4)$ & $120(65.9)$ & \\
\hline Tumor stage & & & & & & 0.007 \\
\hline $\mathrm{T} 1$ & $350(48.5)$ & $167(55.3)$ & $85(50.6)$ & $30(43.5)$ & $68(37.4)$ & \\
\hline $\mathrm{T} 2$ & $356(49.4)$ & $127(42.1)$ & $81(48.2)$ & $38(55.1)$ & $110(60.4)$ & \\
\hline T3 & $15(2.1)$ & $8(2.6)$ & $2(1.2)$ & $1(1.4)$ & $4(2.2)$ & \\
\hline Nodal stage & & & & & & 0.060 \\
\hline NO & $425(58.9)$ & $171(56.6)$ & $92(54.8)$ & $42(60.9)$ & $120(65.9)$ & \\
\hline N1 & $192(26.6)$ & $89(29.5)$ & $43(25.6)$ & $13(18.8)$ & $47(25.8)$ & \\
\hline N2 & $65(9.0)$ & $27(8.9)$ & $18(10.7)$ & $9(13.0)$ & $11(6.0)$ & \\
\hline N3 & $39(5.4)$ & $15(5.0)$ & $15(8.9)$ & $5(7.2)$ & $4(2.2)$ & \\
\hline Estrogen receptor status & & & & & & $<0.001$ \\
\hline Negative & $261(36.2)$ & $5(1.7)$ & $5(3.0)$ & $69(100.0)$ & $182(0.0)$ & \\
\hline Positive & $460(63.8)$ & $297(98.3)$ & $163(97.0)$ & $0(0.0)$ & $0(0.0)$ & \\
\hline Progesterone receptor status & & & & & & $<0.001$ \\
\hline Negative & $346(48.0)$ & $49(16.2)$ & $46(27.4)$ & $69(100.0)$ & $182(100.0)$ & \\
\hline Positive & $375(52.0)$ & $253(83.8)$ & $122(72.6)$ & $0(0.0)$ & $0(0.0)$ & \\
\hline HER-2 status & & & & & & $<0.001$ \\
\hline Negative & $573(79.5)$ & $302(100.0)$ & $89(53.0)$ & $0(0.0)$ & $182(100.0)$ & \\
\hline Positive & $148(20.5)$ & $0(0.0)$ & $79(47.0)$ & $69(100.0)$ & $0(0.0)$ & \\
\hline Ki-67 LI (\%, mean \pm SD) & $17.3 \pm 18.4$ & $4.7 \pm 3.7$ & $19.6 \pm 12.6$ & $19.3 \pm 12.8$ & $35.1 \pm 23.0$ & $<0.001$ \\
\hline Tumor recurrence & $63(8.7)$ & $14(4.6)$ & $13(7.7)$ & $11(15.9)$ & $25(13.7)$ & 0.001 \\
\hline Patient death & $60(8.3)$ & $12(4.0)$ & $13(7.7)$ & $11(15.9)$ & $24(13.2)$ & $<0.001$ \\
\hline $\begin{array}{l}\text { Duration of clinical follow-up } \\
\text { (months, mean } \pm S D)\end{array}$ & $70.0 \pm 31.2$ & $72.4 \pm 29.3$ & $70.2 \pm 30.0$ & $65.2 \pm 34.3$ & $67.8 \pm 34.2$ & 0.234 \\
\hline
\end{tabular}

$\overline{T N B C}$ triple negative breast cancer.

$(-) / \mathrm{SDHB}(-)>\operatorname{SDHA}(+) / \mathrm{SDHB}(+)>\operatorname{SDHA}(-) / \mathrm{SDHB}(+)>$ $\operatorname{SDHA}(+) / \operatorname{SDHB}(-)$ (Table 7$)$.

$\mathrm{SDHA}(+) / \mathrm{SDHB}(+)$ tumors tended to have higher histologic grades than $\mathrm{SDHA}(+) / \mathrm{SDHB}(-)$ and $\mathrm{SDHA}(-) / \mathrm{SDHB}$ $(-)$ tumors $(P=0.038)$. Stromal SDHA $(-) / \mathrm{SDHB}(+)$ tumors showed the highest frequency of HER-2 positivity, while stromal SDHA(+)/SDHB(-) tumors showed lowest HER-2 positivity $(P=0.001)$. Stromal SDHA/SDHB phenotype differed significantly between the molecular subtypes, SDHA $(+) / \mathrm{SDHB}(+)$ being most frequent in Luminal A and least in TNBC tumors. The $\mathrm{SDHA}(+) / \mathrm{SDHB}(-)$ phenotype was most frequent in TNBC and least frequent in HER-2 subtype tumors; $\mathrm{SDHA}(-) / \mathrm{SDHB}(+)$ was most frequent in $\mathrm{Lu}-$ minal $\mathrm{B}$ and least in TNBC; and $\operatorname{SDHA}(-) / \operatorname{SDHB}(-)$ was most frequent in the Luminal $A$ and least in the HER-2 subtype $(P<0.001)$.

\section{Prognostic significance of HIF-1a and SDH expression status}

In univariate analysis, neither HIF- $1 \alpha$ nor SDH expression was significantly related to patient outcome (Table 8). In multivariate Cox analysis (Table 9), factors negatively associated with DFS were T stage (Hazard ratio: 2.535, 95\% CI: $1.376-4.670, P=0.003)$, lymph node metastasis (Hazard ratio: 2.257, 95\% CI: $1.335-3.816, P=0.002$ ), and high histologic grade (Hazard ratio: 1.685, 95\% CI: $1.013-2.804, P=0.045)$. Factors negatively associated with OS were $\mathrm{T}$ stage (Hazard ratio: 2.218, 95\% CI: $1.229-4.001, P=0.008$ ), lymph node metastasis (Hazard ratio: $1.831,95 \% \mathrm{CI}: 1.076-3.115, P=0.026)$, high tumor expression of SDHA (Hazard ratio: 4.157, 95\% CI: 1.657 $10.432, P=0.002$ ), and low SDHB expression (Hazard ratio: 3.223 , 95\% CI: $1.295-8.025, P=0.012$ ). 
Table 3 Expression of HIF-1a and SDH according to tumor phenotype

\begin{tabular}{|c|c|c|c|c|c|c|}
\hline Parameters & Total $n=721(\%)$ & Luminal A $n=302(\%)$ & Luminal B $n=168(\%)$ & HER-2 $n=69$ (\%) & TNBC $n=182(\%)$ & P-value \\
\hline Nuclear HIF-1a & & & & & & 0.319 \\
\hline Negative & $697(96.7)$ & $296(98.0)$ & $161(95.8)$ & $65(94.2)$ & $175(96.2)$ & \\
\hline Positive & $24(3.3)$ & $6(2.0)$ & $7(4.2)$ & $4(5.8)$ & $7(3.8)$ & \\
\hline Cytoplasmic HIF-1a & & & & & & 0.596 \\
\hline Negative & $717(99.4)$ & $301(99.7)$ & $166(98.8)$ & $69(100.0)$ & $181(99.5)$ & \\
\hline Positive & $4(0.6)$ & $1(0.3)$ & $2(1.2)$ & $0(0.0)$ & $1(0.5)$ & \\
\hline Tumoral SDHA & & & & & & 0.032 \\
\hline Negative/Low & 41/319 (49.9) & 21/143 (54.3) & $8 / 76(50.0)$ & $5 / 19(34.8)$ & $7 / 81(48.4)$ & \\
\hline High & $361(50.1)$ & $138(45.7)$ & $84(50.0)$ & $45(65.2)$ & $94(51.6)$ & \\
\hline Stromal SDHA & & & & & & 0.063 \\
\hline Negative & $665(92.2)$ & $286(94.7)$ & $153(91.1)$ & $59(85.5)$ & $167(91.8)$ & \\
\hline Positive & $56(7.8)$ & $16(5.3)$ & $15(8.9)$ & $10(14.5)$ & $15(8.2)$ & \\
\hline Tumoral SDHB & & & & & & 0.291 \\
\hline Negative/Low & $24 / 245(37.3)$ & 15/103 (39.1) & $3 / 56(35.1)$ & 3/19 (31.9) & 3/67 (38.5) & \\
\hline High & $452(62.7)$ & $184(60.9)$ & $109(64.9)$ & $47(68.1)$ & $112(61.5)$ & \\
\hline Stromal SDHB & & & & & & $<0.001$ \\
\hline Negative & $641(88.9)$ & $277(91.7)$ & $142(84.5)$ & 51 (73.9) & $171(94.0)$ & \\
\hline Positive & 80 (11.1) & $25(8.3)$ & $26(15.5)$ & $18(26.1)$ & $11(6.0)$ & \\
\hline
\end{tabular}

TNBC triple negative breast cancer.

\section{Discussion}

Germline defects in SDH, particularly in SDHA and SDHB, have been detected in several tumor types, including pheochromocytomas (Van Nederveen et al. 2009; Astuti et al. 2001), paragangliomas (Van Nederveen et al. 2009; Astuti et al. 2001; Baysal 2003; Burnichon et al. 2010), GISTs (Gaal et al. 2011; Gill et al. 2010b; Gill et al. 2011a), and renal cell carcinomas (Gill et al. 2011b). Until recently, the accurate detection of SDH mutation depended primarily on direct sequencing and western blotting, methods that a clinical laboratory may find too costly and timeconsuming. However, in a study of paraganglioma and pheochromocytoma, Van Nederveen et al. demonstrated the high sensitivity of immunohistochemical

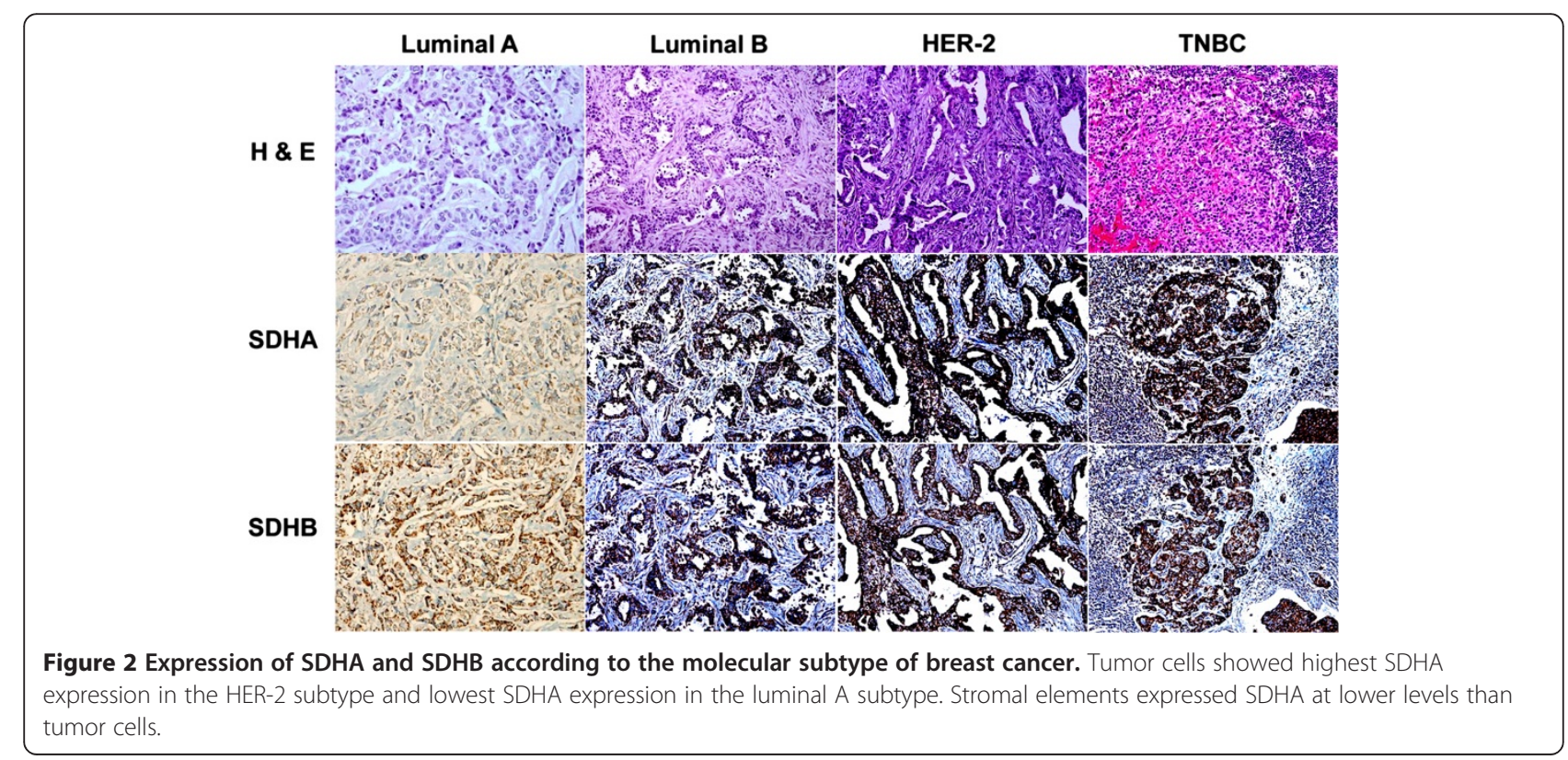


Table 4 Expression of SDHA and SDHB according to HIF-1a expression status

\begin{tabular}{|c|c|c|c|c|c|c|}
\hline \multirow[t]{2}{*}{ Factors } & \multicolumn{3}{|c|}{ Nuclear HIF-1a } & \multicolumn{3}{|c|}{ Cytoplasmic HIF-1a } \\
\hline & Negative $n=697(\%)$ & Positive $n=24(\%)$ & $P$-value & Negative $n=717(\%)$ & Positive $n=4(\%)$ & $P$-value \\
\hline Tumoral SDHA & & & 0.247 & & & 0.518 \\
\hline Negative & $40(5.7)$ & $1(4.2)$ & & $40(5.6)$ & $1(25.0)$ & \\
\hline Low & $311(44.6)$ & $8(33.3)$ & & $318(44.4)$ & $1(25.0)$ & \\
\hline High & $346(49.6)$ & $15(62.5)$ & & $359(50.1)$ & $50.0)$ & \\
\hline Stromal SDHA & & & 0.423 & & & 1.000 \\
\hline Negative & $644(92.4)$ & $21(87.5)$ & & $661(92.2)$ & $4(100.0)$ & \\
\hline Positive & $53(7.6)$ & $3(12.5)$ & & $56(7.8)$ & $0(0.0)$ & \\
\hline Tumoral SDHB & & & 0.926 & & & 0.032 \\
\hline Negative & $24(3.4)$ & $0(0.0)$ & & $23(3.2)$ & $1(25.0)$ & \\
\hline Low & $235(33.7)$ & $10(41.7)$ & & $243(33.9)$ & $2(50.0)$ & \\
\hline High & $438(62.8)$ & $14(58.3)$ & & $451(62.9)$ & $1(25.0)$ & \\
\hline Stromal SDHB & & & 0.328 & & & 1.000 \\
\hline Negative & $621(89.1)$ & $20(83.3)$ & & $637(88.8)$ & $4(100.0)$ & \\
\hline Positive & $76(10.9)$ & $4(16.7)$ & & $80(11.2)$ & $0(0.0)$ & \\
\hline
\end{tabular}

SDH succinate dehydrogenase.

methods to detect germline mutations in SDH (Van Nederveen et al. 2009). While the tumors with SDHB, SDHC, or SDHD germline mutations exhibited a loss of SDHB immmunoexpression with intact SDHA expression, tumors with SDHA germline mutations exhibited a loss of expression of both SDHA and SDHB.
Using similar methods in the present study, we found that 23 of 721 breast cancer patients (3.19\%) had SDHA mutation (SDHA-/SDHB- expression) and one patient (0.1\%) had an SDHB mutation (SHDA+/SDHB- expression; Table 7). As few previous studies have evaluated $\mathrm{SDH}$ mutation in breast cancer, these findings provide a starting point for future investigations. However, a

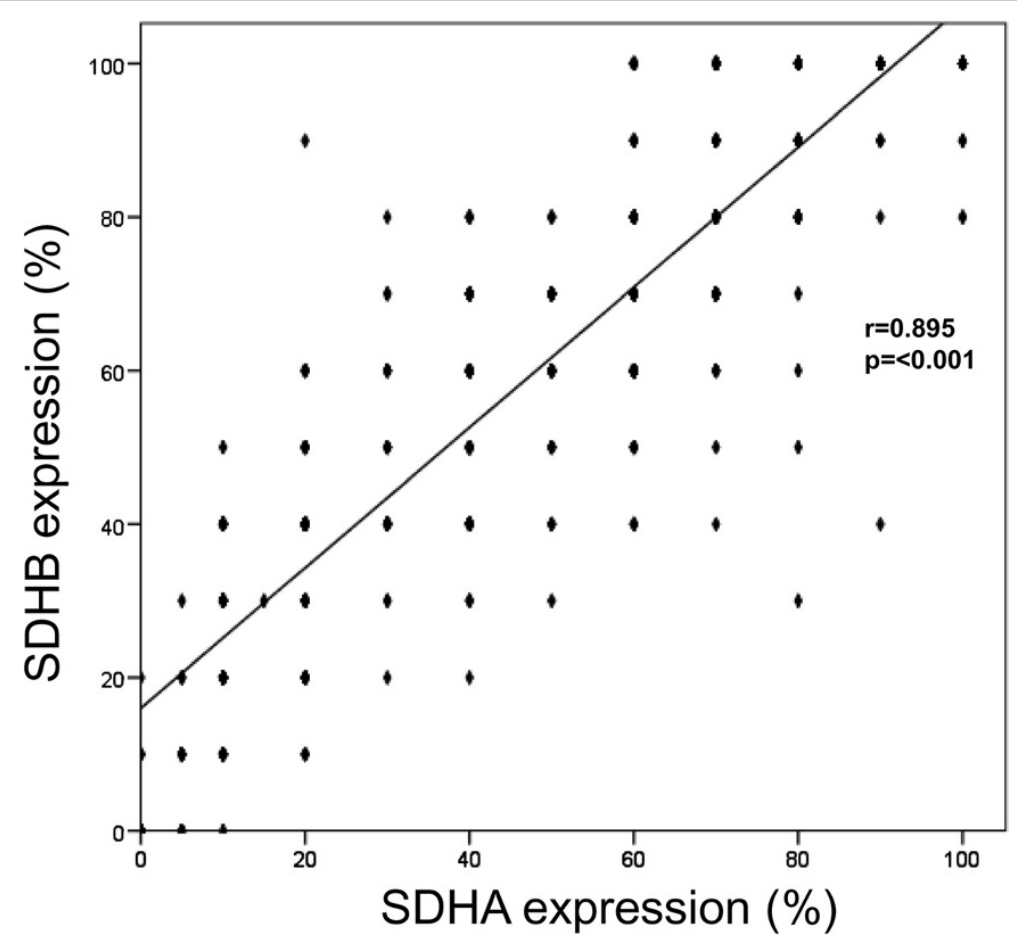

Figure 3 Correlation between SDHA and SDHB expression. 
Table 5 Clinicopathologic characteristics of breast cancer with SDHA and/or SDHB negativity in tumor cells

\begin{tabular}{|c|c|c|c|c|c|c|}
\hline \multirow[t]{2}{*}{ Parameters } & \multicolumn{3}{|c|}{ SDHA } & \multicolumn{3}{|c|}{ SDHB } \\
\hline & Negative $n=41(\%)$ & Positive $n=680(\%)$ & $P$-value & Negative $n=24(\%)$ & Positive $n=697(\%)$ & $P$-value \\
\hline Age (years, mean $\pm S D$ ) & $45.4 \pm 10.3$ & $49.9 \pm 10.9$ & 0.012 & $46.9 \pm 9.1$ & $49.7 \pm 10.9$ & 0.212 \\
\hline Histologic grade & & & 0.062 & & & 0.044 \\
\hline$|/| \mid$ & $33(80.5)$ & $451(66.3)$ & & $21(87.5)$ & $463(66.4)$ & \\
\hline III & $8(19.5)$ & $229(33.7)$ & & $3(12.5)$ & $234(33.6)$ & \\
\hline ER & & & 0.405 & & & 0.286 \\
\hline Negative & $12(29.3)$ & $249(36.6)$ & & $6(25.0)$ & $255(36.6)$ & \\
\hline Positive & $29(70.7)$ & $431(63.4)$ & & $18(75.0)$ & $442(63.4)$ & \\
\hline PR & & & 0.748 & & & 1.000 \\
\hline Negative & $21(51.2)$ & $325(47.8)$ & & $11(45.8)$ & $335(48.1)$ & \\
\hline Positive & $20(48.8)$ & $355(52.2)$ & & $13(54.2)$ & $362(51.9)$ & \\
\hline HER-2 & & & 0.693 & & & 0.800 \\
\hline Negative & $34(82.9)$ & $539(79.3)$ & & $20(83.3)$ & $553(79.3)$ & \\
\hline Positive & $7(17.1)$ & $141(20.7)$ & & $4(16.7)$ & $144(20.7)$ & \\
\hline Tumor stage & & & 0.750 & & & 0.213 \\
\hline $\mathrm{T} 1$ & $21(51.2)$ & $329(48.4)$ & & $15(62.5)$ & $335(48.1)$ & \\
\hline $\mathrm{T} 2 / \mathrm{T} 3$ & $20(48.8)$ & $351(51.6)$ & & $9(37.5)$ & $362(51.9)$ & \\
\hline Nodal stage & & & 0.625 & & & 0.529 \\
\hline No & $26(63.4)$ & $399(58.7)$ & & $16(66.7)$ & $409(58.7)$ & \\
\hline $\mathrm{N} 1 / \mathrm{N} 2 / \mathrm{N} 3$ & 15 (36.6) & $281(41.3)$ & & $8(33.3)$ & $288(41.3)$ & \\
\hline Molecular subtypes & & & 0.452 & & & 0.134 \\
\hline Luminal A & $21(51.2)$ & $281(41.3)$ & & $15(62.5)$ & $287(41.2)$ & \\
\hline Luminal B & $8(19.5)$ & $160(23.5)$ & & $3(12.5)$ & $165(23.7)$ & \\
\hline HER-2 & $5(12.2)$ & $64(9.4)$ & & $3(12.5)$ & $66(9.5)$ & \\
\hline TNBC & $7(17.1)$ & $175(25.7)$ & & $3(12.5)$ & $179(25.7)$ & \\
\hline Ki-67 LI (\%, mean \pm SD) & $13.0 \pm 12.3$ & $17.5 \pm 18.7$ & 0.127 & $9.9 \pm 10.0$ & $17.5 \pm 18.6$ & 0.046 \\
\hline Tumor recurrence & $4(9.8)$ & $59(8.7)$ & 0.775 & $1(4.2)$ & $62(8.9)$ & 0.714 \\
\hline Patient death & $4(9.8)$ & $56(8.2)$ & 0.768 & $1(4.2)$ & $59(8.5)$ & 0.713 \\
\hline
\end{tabular}

$S D H$ succinate dehydrogenase, $T N B C$ triple negative breast cancer.

previous study reported that SDH germline mutations or variants occur in some patients with Cowden syndrome (CS) who do not present the expected PTEN mutation. Compared with patients positive for germline PTEN mutation, these CS/CS-like individuals develop cancers of the breast, thyroid, and kidney at higher frequencies (Ni et al. 2008). Therefore, some breast cancer patients may be expected to have SDH mutations.

Findings in this study may be limited in that the sensitivity of immunohistochemistry in detecting SDH mutation as compared to direct sequencing has not been tested in breast cancer. We have assumed a degree of reliability in breast cancer similar to that of the detection of $\mathrm{SDH}$ germline mutations in paraganglioma and pheochromocytoma (Van Nederveen et al. 2009). To confirm SDH mutation, loss of SDH expression should be tested throughout the entire tumor (Barletta \& Hornick 2012), whereas in this study, immunohistochemistry was performed on the tissue microarray only.

In previous studies of pheochromocytoma and GIST, SDH-deficient tumors showed complete loss of cytoplasmic granular expression (Miettinen et al. 2011; GimenezRoqueplo et al. 2003). Even though weak, focal or diffuse cytoplasmic staining was observed in a very few tumor cells of SDH-deficient tumor, this finding may be considered negative, because it is clearly distinguishable from the strong speckled expression pattern in surrounding non-neoplastic elements. However, stromal cells did not express SDHA and SDHB in most breast cancers in this study, which made it impossible to compare the staining intensity between tumor cells and internal normal controls (stromal cells). Therefore, expression in tumor cells was interpreted at three grade levels according to the proportion of cells stained: grade (1), 
Table 6 Clinicopathologic characteristics breast cancer by expression of HIF-1a and SDH

\begin{tabular}{|c|c|c|c|c|c|c|c|c|c|c|c|c|c|c|c|c|c|c|}
\hline \multirow[t]{2}{*}{ Parameters } & \multicolumn{3}{|c|}{ Nuclear HIF-1a } & \multicolumn{3}{|c|}{ Cytoplasmic HIF-1a } & \multicolumn{3}{|c|}{ Tumor SDHA } & \multicolumn{3}{|c|}{ Stromal SDHA } & \multicolumn{3}{|c|}{ Tumor SDHB } & \multicolumn{3}{|c|}{ Stromal SDHB } \\
\hline & - & + & $P$ & - & + & $P$ & $-*$ & + & $P$ & - & + & $P$ & $-*$ & + & $P$ & - & + & $P$ \\
\hline Histologic grade & & & 0.270 & & & 0.601 & & & 0.342 & & & 0.105 & & & 0.870 & & & 0.528 \\
\hline |//1 & $465(66.7)$ & $19(79.2)$ & & $482(67.2)$ & $2(50.0)$ & & $248(68.9)$ & $236(65.4)$ & & $452(68.0)$ & $32(57.1)$ & & $182(67.7)$ & $302(66.8)$ & & $433(67.6)$ & $51(63.8)$ & \\
\hline III & $232(33.3)$ & $5(20.8)$ & & $235(32.8)$ & $2(50.0)$ & & $112(31.2)$ & $125(34.6)$ & & $213(32.0)$ & $24(42.9)$ & & 87 (32.3) & $150(33.2)$ & & $208(32.4)$ & $29(36.3)$ & \\
\hline ER & & & 0.388 & & & 1.000 & & & 0.044 & & & 0.111 & & & 0.873 & & & 0.806 \\
\hline Negative & $250(35.9)$ & $11(45.8)$ & & $260(36.3)$ & $1(25.0)$ & & $117(32.5)$ & $144(39.9)$ & & $235(35.3)$ & $26(46.4)$ & & $96(35.7)$ & $165(36.5)$ & & $231(36.0)$ & $30(37.5)$ & \\
\hline Positive & $447(64.1)$ & $13(54.2)$ & & $457(63.7)$ & $3(75.0)$ & & $243(67.5)$ & $217(60.1)$ & & $430(64.7)$ & $30(53.6)$ & & $173(64.3)$ & $287(63.5)$ & & $410(64.0)$ & $50(62.5)$ & \\
\hline$P R$ & & & 0.011 & & & 1.000 & & & 0.118 & & & 0.165 & & & 0.645 & & & 1.000 \\
\hline Negative & $328(47.1)$ & 18 (75.0) & & $344(48.0)$ & $2(50.0)$ & & $162(45.0)$ & $184(51.0)$ & & $314(47.2)$ & $32(57.1)$ & & $126(46.8)$ & $220(48.7)$ & & $308(48.0)$ & $38(47.5)$ & \\
\hline Positive & $369(52.9)$ & $6(25.0)$ & & $373(52.0)$ & $2(50.0)$ & & $198(55.0)$ & $177(49.0)$ & & $351(52.8)$ & $24(42.9)$ & & $143(53.2)$ & $232(51.3)$ & & $333(52.0)$ & $42(52.5)$ & \\
\hline HER-2 & & & 0.304 & & & 1.000 & & & 0.021 & & & 0.083 & & & 0.057 & & & $<0.001$ \\
\hline Negative & $556(79.8)$ & 17 (70.8) & & $570(79.5)$ & $3(75.0)$ & & $299(83.1)$ & $274(75.9)$ & & $534(80.3)$ & 39 (69.6) & & $224(83.3)$ & $349(77.2)$ & & $526(82.1)$ & $47(58.8)$ & \\
\hline Positive & $141(20.2)$ & 7 (29.2) & & $147(20.5)$ & $1(25.0)$ & & $61(16.9)$ & $87(24.1)$ & & 131 (19.7) & 17 (30.4) & & 45 (16.7) & $103(22.8)$ & & 115 (17.9) & $33(41.3)$ & \\
\hline Tumor stage & & & 0.304 & & & 1.000 & & & 0.031 & & & 1.000 & & & 0.011 & & & 0.237 \\
\hline $\mathrm{T} 1$ & $341(48.9)$ & $9(37.5)$ & & $348(48.5)$ & $2(50.0)$ & & $160(44.4)$ & 190 (52.6) & & 323 (48.6) & $27(48.2)$ & & $114(42.4)$ & $236(52.2)$ & & $306(47.7)$ & $44(55.0)$ & \\
\hline $\mathrm{T} 2 / \mathrm{T} 3$ & $356(51.1)$ & $15(62.5)$ & & $369(51.5)$ & $2(50.0)$ & & 200 (55.6) & $171(47.4)$ & & $342(51.4)$ & 29 (51.8) & & $155(57.6)$ & $216(47.8)$ & & $335(52.3)$ & $36(45.0)$ & \\
\hline Nodal stage & & & 0.208 & & & 0.648 & & & 0.198 & & & 0.575 & & & 0.639 & & & 0.149 \\
\hline NO & $414(59.4)$ & $11(45.8)$ & & $422(58.9)$ & $3(75.0)$ & & $221(61.4)$ & $204(56.5)$ & & $394(59.2)$ & $31(55.4)$ & & $162(60.2)$ & $263(58.2)$ & & $384(59.9)$ & $41(51.3)$ & \\
\hline N1/N2/N3 & $283(40.6)$ & $13(54.2)$ & & $295(41.1)$ & $1(25.0)$ & & 139 (38.6) & $157(43.5)$ & & 271 (40.8) & 25 (44.6) & & 107 (39.8) & $189(41.8)$ & & $257(40.1)$ & 39 (48.8) & \\
\hline
\end{tabular}

SDH succinate dehydrogenase.

*includes negative and low-positive cases. 
Table 7 Clinicopathologic features according to the SDH expression phenotype in breast cancer

\begin{tabular}{|c|c|c|c|c|c|c|c|c|c|c|}
\hline \multirow[t]{2}{*}{ Factors } & \multicolumn{5}{|c|}{ Tumor phenotype } & \multicolumn{5}{|c|}{ Stroma phenotype } \\
\hline & $\begin{array}{l}\text { SDHA (+)/ SDHB } \\
(+) n=679(\%)\end{array}$ & $\begin{array}{c}\text { SDHA (+)/ SDHB } \\
\quad(-) n=1 \text { (\%) }\end{array}$ & $\begin{array}{c}\text { SDHA (-)/ SDHB } \\
(+) n=18(\%)\end{array}$ & $\begin{array}{c}\text { SDHA (-)/ SDHB } \\
(-) n=23(\%)\end{array}$ & $P$ & $\begin{array}{c}\text { SDHA (+)/ SDHB } \\
(+) n=42(\%)\end{array}$ & $\begin{array}{c}\text { SDHA (+)/ SDHB } \\
(-) n=14(\%)\end{array}$ & $\begin{array}{c}\text { SDHA (-)/ SDHB } \\
(+) n=38(\%)\end{array}$ & $\begin{array}{c}\text { SDHA (-)/ SDHB } \\
(-) n=627(\%)\end{array}$ & $P$ \\
\hline Histologic grade & & & & & 0.038 & & & & & 0.150 \\
\hline$|/| \mid$ & $450(66.3)$ & $1(100.0)$ & $13(72.2)$ & $20(87.0)$ & & $25(59.5)$ & $7(50.0)$ & $26(68.4)$ & $426(67.9)$ & \\
\hline III & $229(33.7)$ & $0(0.0)$ & $5(27.8)$ & $3(13.0)$ & & $17(40.5)$ & $7(50.0)$ & $12(31.6)$ & $201(32.1)$ & \\
\hline Tumor stage & & & & & 0.495 & & & & & 0.640 \\
\hline $\mathrm{T} 1$ & $329(48.5)$ & $0(0.0)$ & $6(33.3)$ & $15(65.2)$ & & $20(47.6)$ & $7(50.0)$ & $24(63.2)$ & $299(47.7)$ & \\
\hline $\mathrm{T} 2 / \mathrm{T} 3$ & $350(51.5)$ & $1(100.0)$ & $12(66.7)$ & $8(34.8)$ & & $22(52.4)$ & $7(50.0)$ & $14(36.8)$ & $328(52.3)$ & \\
\hline Nodal stage & & & & & 0.491 & & & & & 0.314 \\
\hline No & $398(58.6)$ & $1(100.0)$ & $11(61.1)$ & $15(65.2)$ & & $21(50.0)$ & $10(71.4)$ & $20(52.6)$ & $374(59.6)$ & \\
\hline N1/N2/N3 & $281(41.4)$ & $0(0.0)$ & $7(38.9)$ & $8(34.8)$ & & $21(50.0)$ & $4(28.6)$ & $18(47.4)$ & $253(40.4)$ & \\
\hline ER & & & & & 0.284 & & & & & 0.205 \\
\hline Negative & $248(36.5)$ & $1(100.0)$ & $7(38.9)$ & $5(21.7)$ & & $16(38.1)$ & $10(71.4)$ & $14(36.8)$ & $221(35.2)$ & \\
\hline Positive & $431(63.5)$ & $0(0.0)$ & $11(61.1)$ & $18(78.3)$ & & $26(61.9)$ & $4(28.6)$ & $24(63.2)$ & $406(64.8)$ & \\
\hline PR & & & & & 0.793 & & & & & 0.336 \\
\hline Negative & $324(47.7)$ & $1(100.0)$ & $11(61.1)$ & $10(43.5)$ & & $21(50.0)$ & $11(78.6)$ & $17(44.7)$ & $297(47.4)$ & \\
\hline Positive & $355(52.3)$ & $0(0.0)$ & $7(38.9)$ & $13(56.5)$ & & $21(50.0)$ & $3(21.4)$ & $21(55.3)$ & $330(52.6)$ & \\
\hline HER-2 & & & & & 0.567 & & & & & 0.001 \\
\hline Negative & $539(79.4)$ & $0(0.0)$ & $14(77.8)$ & $20(87.0)$ & & $26(61.9)$ & $13(92.9)$ & $21(55.3)$ & $513(81.8)$ & \\
\hline Positive & $140(20.6)$ & $1(100.0)$ & $4(22.2)$ & $3(13.0)$ & & $16(38.1)$ & $1(7.1)$ & $17(44.7)$ & $114(18.2)$ & \\
\hline Molecular type & & & & & 0.057 & & & & & $<0.001$ \\
\hline Luminal A & $281(41.4)$ & $0(0.0)$ & $6(33.3)$ & $15(65.2)$ & & $14(33.3)$ & $2(14.3)$ & $11(28.9)$ & $275(43.9)$ & \\
\hline Luminal B & $160(23.6)$ & $0(0.0)$ & $5(27.8)$ & $3(13.0)$ & & $13(31.0)$ & $2(14.3)$ & $13(34.2)$ & $140(22.3)$ & \\
\hline HER-2 & $63(9.3)$ & $1(100.0)$ & $3(16.7)$ & $2(8.7)$ & & $9(21.4)$ & $1(7.1)$ & $9(23.7)$ & $50(8.0)$ & \\
\hline TNBC & $175(25.8)$ & $0(0.0)$ & $4(22.2)$ & $3(13.0)$ & & $6(14.3)$ & $9(64.3)$ & $5(13.2)$ & $162(25.8)$ & \\
\hline Ki-67 LI (\%, mean \pm SD) & $17.5 \pm 18.7$ & $8.0 \pm 0.0$ & $16.8 \pm 14.0$ & $10.0 \pm 10.2$ & 0.260 & $17.8 \pm 15.3$ & $24.3 \pm 22.8$ & $22.1 \pm 19.0$ & $16.8 \pm 18.4$ & 0.161 \\
\hline Tumor recurrence & $59(8.7)$ & $0(0.0)$ & $3(16.7)$ & $1(4.3)$ & 0.955 & $4(9.5)$ & $1(7.1)$ & $2(5.3)$ & $56(8.9)$ & 0.877 \\
\hline Patient death & $56(8.2)$ & $0(0.0)$ & $3(16.7)$ & $1(4.3)$ & 0.968 & $3(7.1)$ & $1(7.1)$ & $3(7.9)$ & $53(8.5)$ & 0.727 \\
\hline
\end{tabular}

$S D H$ succinate dehydrogenase. 
Table 8 Univariate analysis of disease-free survival and overall survival according to breast-tumor expression of hypoxia-related proteins

\begin{tabular}{|c|c|c|c|c|c|}
\hline \multirow[t]{2}{*}{ Parameters } & \multirow{2}{*}{$\begin{array}{l}\text { Number of patients/ } \\
\text { recurrence/death }\end{array}$} & \multicolumn{2}{|c|}{ Disease-free survival } & \multicolumn{2}{|c|}{ Overall survival } \\
\hline & & $\begin{array}{l}\text { Mean survival } \\
(95 \% \mathrm{Cl}) \text { months }\end{array}$ & $P$-value & $\begin{array}{l}\text { Mean survival } \\
(95 \% \mathrm{Cl}) \text { months }\end{array}$ & $P$-value \\
\hline Nuclear HIF-1a & & & 0.911 & & 0.943 \\
\hline Negative & $697 / 61 / 58$ & $126(122-130)$ & & $129(127-132)$ & \\
\hline Positive & $24 / 2 / 2$ & $120(108-133)$ & & $131(122-141)$ & \\
\hline Cytoplasmic HIF-1a & & & N/A & & N/A \\
\hline Negative & $717 / 63$ & N/A & & N/A & \\
\hline Positive & $4 / 0$ & N/A & & N/A & \\
\hline Tumoral SDHA & & & 0.670 & & 0.085 \\
\hline Negative/Low & $360 / 35 / 26$ & $125(121-129)$ & & $131(128-135)$ & \\
\hline High & $361 / 28 / 34$ & $126(120-132)$ & & $126(121-130)$ & \\
\hline Stromal SDHA & & & 0.853 & & 0.901 \\
\hline Negative & $665 / 58 / 56$ & $126(123-130)$ & & $130(127-132)$ & \\
\hline Positive & $56 / 5 / 4$ & $115(101-130)$ & & $121(107-134)$ & \\
\hline Tumoral SDHB & & & 0.124 & & 0.715 \\
\hline Negative/Low & 269/31/26 & $123(119-128)$ & & $129(125-133)$ & \\
\hline High & $452 / 32 / 34$ & $126(121-131)$ & & $129(125-132)$ & \\
\hline Stromal SDHB & & & 0.821 & & 0.981 \\
\hline Negative & $641 / 57 / 54$ & $126(122-130)$ & & $130(127-132)$ & \\
\hline Positive & $80 / 6 / 6$ & $119(110-128)$ & & $123(114-131)$ & \\
\hline
\end{tabular}

$S D H$ succinate dehydrogenase $\mathrm{P}$-value by log-rank test.

Table 9 Multivariate analysis for breast-cancer survival

\begin{tabular}{|c|c|c|c|c|c|c|}
\hline \multirow[t]{2}{*}{ Parameters } & \multicolumn{3}{|c|}{ Disease-free survival } & \multicolumn{3}{|c|}{ Overall survival } \\
\hline & Hazard ratio & $95 \% \mathrm{Cl}$ & $P$-value & Hazard ratio & $95 \% \mathrm{Cl}$ & $P$-value \\
\hline Tstage & & & 0.003 & & & 0.008 \\
\hline T1 versus T2-3 & 2.535 & $1.376-4.670$ & & 2.218 & $1.229-4.001$ & \\
\hline N stage & & & 0.002 & & & 0.026 \\
\hline No versus N1-3 & 2.257 & $1.335-3.816$ & & 1.831 & $1.076-3.115$ & \\
\hline Histologic grade & & & 0.045 & & & 0.372 \\
\hline$|/| \mid$ versus III & 1.685 & $1.013-2.804$ & & 1.276 & $0.748-2.176$ & \\
\hline Nuclear HIF-1a & & & 0.599 & & & 0.486 \\
\hline Negative versus Positive & 0.682 & $0.163-2.846$ & & 0.599 & $0.142-2.528$ & \\
\hline Tumor SDHA & & & 0.290 & & & 0.002 \\
\hline Negative/Low versus High & 1.570 & $0.681-3.620$ & & 4.157 & $1.657-10.432$ & \\
\hline Stromal SDHA & & & 0.727 & & & 0.747 \\
\hline Negative versus Positive & 1.254 & $0.352-4.468$ & & 0.807 & $0.218-2.978$ & \\
\hline Tumor SDHB & & & 0.096 & & & 0.012 \\
\hline Negative/Low versus High & 0.498 & $0.219-1.131$ & & 3.223 & $1.295-8.025$ & \\
\hline Stromal SDHB & & & 0.712 & & & 0.965 \\
\hline Negative versus Positive & 0.802 & $0.248-2.590$ & & 0.976 & $0.327-2.908$ & \\
\hline
\end{tabular}

SDH succinate dehydrogenase. 
negative (complete loss of expression); grade (2), low (expression in less than $50 \%$ of cells); and grade (3) high (expression in more than $50 \%$ of cells).

Several mechanisms have been proposed to explain the involvement of SDH mutation in tumorigenesis, among which a HIF-1 $\alpha$-pathway-dependent mechanism is the most famous. Loss-of-function mutation of SDH could result in an intracellular SDH accumulation, which in turn inhibits prolyl 4-hydroxylase (PHD), a negative regulator of HIF-1 $\alpha$ (Cardaci \& Ciriolo 2012; Barletta \& Hornick 2012). The impaired PHD activity stabilizes HIF- $1 \alpha$ under normoxic condition, which upregulates HIF target gene involved in cell growth stimulation and angiogenesis, thus contributing to tumor progression. As loss-of-function mutations in SDH are predicted to stabilize HIF- $1 \alpha$ and upregulate HIF-1 transcriptional activity, we examined the expression of HIF- $1 \alpha$ along with SDHA/SDHB. However, we found no close relationship between HIF-1 $\alpha$ and SDH expression in these breast cancers.

SDH-deficient GISTs and renal cell carcinomas display characteristic histologic features distinguishable from tumors without SDH mutation (Miettinen et al. 2011; Gill et al. 2011b). Similarly, the SDHA+/SDHB- and SDHA/SDHB- breast cancers in this study were distinguished by lower histologic grade (Table 7), and in the SDHB-negative tumors, by lower Ki-67 LI (Table 5). The luminal A breast cancer subtype showed the highest frequency of low/negative SDHA expression ( $P=0.032$, Table 3$)$. Based on these results, $\mathrm{SDH}$ mutation in breast cancer is associated with low histologic grade and a less aggressive molecular subtype.

Patients with SDHA- and SDHB-negative breast cancers were also younger than patients with intact $\mathrm{SDH}$ expression, although in the case of SDHB, this difference was not statistically significant. In earlier studies of SDH mutations in renal cell carcinomas (Baysal 2003), GISTs (Miettinen et al. 2011), paragangliomas, and pheochromocytomas (Gimenez-Roqueplo et al. 2003), tumors also occurred at younger ages.

Metabolism in many malignant tumors is characterized by the Warburg effect, which is a high level of anaerobic glucose metabolism by glycolysis despite presence of oxygen with relatively low mitochondrial oxidative phosphorylation (OXPHOS) (Warburg 1956). In this study, more than 50\% of breast cancers expressed SDHA and SDHB, which are key components of aerobic glucose metabolism through the TCA cycle and mitochondrial electron transport. This finding of high mitochondrial activity in breast cancer agrees with an earlier report that breast cancer cells show high expression of mitochondrial metabolic enzymes such as cytochrome $\mathrm{C}$ oxidase, $\mathrm{NADH}$, and SDHB (Whitaker-Menezes et al. 2011).

Expression of Glut-1 and CAIX (indicators of glycolysis) has also been observed in breast cancers, most notably in high-grade tumors such as TNBC or basallike carcinoma (Choi et al. 2013; Pinheiro et al. 2011). While breast cancers overall may express high levels of activity in both OXPHOS and glycolysis, the predominant mode of energy metabolism may differ according to the tumor type (Moreno-Sanchez et al. 2007: Kallinowski et al. 1989; Liu et al. 2001).

Although most breast cancers in this study were negative for SDH in stromal elements, stromal SDHB expression differed significantly among the molecular subtypes. The HER-2 subtype showed the highest and TNBC showed the lowest frequency of stromal SDHB expression (Table 3). However, in a previous study, stromal cells in breast cancer did not express mitochondrial metabolic enzymes (cytochrome c oxidase, NADH, or SDHB) (Whitaker-Menezes et al. 2011). Further studies will be required to determine the differences in stromal SDHB expression among breast cancer molecular subtypes.

In conclusion, loss of SDHA or SDHB expression was detected in approximately $3 \%$ of the breast cancers in this study. Patients with SDH-deficient breast cancers were younger at diagnosis and presented tumors of relatively low-grade histology.

\section{Consent}

Written informed consent was obtained from the patient for the publication of this report and any accompanying images.

\section{Competing interest}

The authors declare no conflict of interest.

\section{Authors' contributions}

SK participated in the design of the study and performed the statistical analysis and drafted the manuscript. DHK carried out the immunoassays. WHJ participated in its design. JSK conceived of the study, and participated in its design and coordination and helped to draft the manuscript. All authors read and approved the final manuscript.

\section{Acknowledgements}

This research was supported by Basic Science Research Program through the National Research Foundation of Korea (NRF), funded by the Ministry of Education, Science and Technology (2012R1A1A1002886).

Received: 10 June 2013 Accepted: 20 June 2013

Published: 3 July 2013

\footnotetext{
References

Anderson BO, Lawton TJ, Lehman CD, Moe RE (2004) Phyllodes tumor. In: Harris JR, Lippman ME, Morrow M, Osborne KC (eds) Disease of the Breast, 3rd edn. Lippincott \& Wilkins, Philadelphia, pp 991-1006

Astuti D, Latif F, Dallol A, Dahia PL, Douglas F, George E, Skoldberg F, Husebye ES, Eng C, Maher ER (2001) Gene mutations in the succinate dehydrogenase subunit SDHB cause susceptibility to familial pheochromocytoma and to familial paraganglioma. Am J Hum Genet 69:49-54. doi:10.1086/321282 Badve S, Dabbs DJ, Schnitt SJ, Baehner FL, Decker T, Eusebi V, Fox SB, Ichihara S, Jacquemier J, Lakhani SR, Palacios J, Rakha EA, Richardson AL, Schmitt FC, Tan PH, Tse GM, Weigelt B, Ellis IO, Reis-Filho JS (2011) Basal-like and triplenegative breast cancers: a critical review with an emphasis on the implications for pathologists and oncologists. Mod Pathol 24:157-167. doi:10.1038/modpathol.2010.200
} 
Barletta JA, Hornick JL (2012) Succinate dehydrogenase-deficient tumors: diagnostic advances and clinical implications. Adv Anat Pathol 19:193-203. doi:10.1097/PAP.0b013e31825c6bc6

Baysal BE (2003) On the association of succinate dehydrogenase mutations with hereditary paraganglioma. Trends Endocrinol Metab 14:453-459

Burnichon N, Briere JJ, Libe R, Vescovo L, Riviere J, Tissier F, Jouanno E, Jeunemaitre X, Benit P, Tzagoloff A, Rustin P, Bertherat J, Favier J, GimenezRoqueplo AP (2010) SDHA is a tumor suppressor gene causing paraganglioma. Hum Mol Genet 19:3011-3020. doi:10.1093/hmg/ddq206

Cardaci S, Ciriolo MR (2012) TCA cycle defects and cancer: when metabolism tunes redox state. Int J Cell Biol 2012:161837. doi:10.1155/2012/161837

Choi J, Jung WH, Koo JS (2013) Metabolism-related proteins are differentially expressed according to the molecular subtype of invasive breast cancer defined by surrogate immunohistochemistry. Pathobiology 80:41-52. doi:10.1159/000339513

Elston CW, Ellis IO (1991) Pathological prognostic factors in breast cancer. I. The value of histological grade in breast cancer: experience from a large study with long-term follow-up. Histopathology 19:403-410

Gaal J, Stratakis CA, Carney JA, Ball ER, Korpershoek E, Lodish MB, Levy I, Xekouki P, Van Nederveen FH, Den Bakker MA, O'Sullivan M, Dinjens WN, De Krijger RR (2011) SDHB immunohistochemistry: a useful tool in the diagnosis of CarneyStratakis and Carney triad gastrointestinal stromal tumors. Mod Pathol 24:147-151. doi:10.1038/modpathol.2010.185

Gill AJ, Benn DE, Chou A, Clarkson A, Muljono A, Meyer-Rochow GY, Richardson AL, Sidhu SB, Robinson BG, Clifton-Bligh RJ (2010a) Immunohistochemistry for SDHB triages genetic testing of SDHB, SDHC, and SDHD in paragangliomapheochromocytoma syndromes. Hum Pathol 41:805-814. doi:10.1016/j. humpath.2009.12.005

Gill AJ, Chou A, Vilain R, Clarkson A, Lui M, Jin R, Tobias V, Samra J, Goldstein D, Smith C, Sioson L, Parker N, Smith RC, Sywak M, Sidhu SB, Wyatt JM, Robinson BG, Eckstein RP, Benn DE, Clifton-Bligh RJ (2010b) Immunohistochemistry for SDHB divides gastrointestinal stromal tumors (GISTs) into 2 distinct types. Am J Surg Pathol 34:636-644. doi:10.1097/ PAS.0b013e3181d6150d

Gill AJ, Chou A, Vilain RE, Clifton-Bligh RJ (2011a) "Pediatric-type" gastrointestinal stromal tumors are SDHB negative ("type 2") GISTs. Am J Surg Pathol 35:1245-1247. doi:10.1097/PAS.0b013e3182217b93

Gill AJ, Pachter NS, Chou A, Young B, Clarkson A, Tucker KM, Winship IM, Earls P, Benn DE, Robinson BG, Fleming S, Clifton-Bligh RJ (2011b) Renal tumors associated with germline SDHB mutation show distinctive morphology. Am J Surg Pathol 35:1578-1585. doi:10.1097/PAS.0b013e318227e7f4

Gimenez-Roqueplo AP, Favier J, Rustin P, Rieubland C, Crespin M, Nau V, Khau Van Kien P, Corvol P, Plouin PF, Jeunemaitre X, Network C (2003) Mutations in the SDHB gene are associated with extra-adrenal and/or malignant phaeochromocytomas. Cancer Res 63:5615-5621

Goldhirsch A, Wood WC, Coates AS, Gelber RD, Thurlimann B, Senn HJ, Panel M (2011) Strategies for subtypes--dealing with the diversity of breast cancer: highlights of the St. Gallen International Expert Consensus on the Primary Therapy of Early Breast Cancer 2011. Ann Oncol 22:1736-1747. doi:10.1093/ annonc/mdr304

Hammond ME, Hayes DF, Dowsett M, Allred DC, Hagerty KL, Badve S, Fitzgibbons PL, Francis G, Goldstein NS, Hayes M, Hicks DG, Lester S, Love R, Mangu PB, McShane L, Miller K, Osborne CK, Paik S, Perlmutter J, Rhodes A, Sasano H, Schwartz JN, Sweep FC, Taube S, Torlakovic EE, Valenstein P, Viale G, Visscher D, Wheeler T, Williams RB, Wittliff JL, Wolff AC (2010) American Society of Clinical Oncology/College Of American Pathologists guideline recommendations for immunohistochemical testing of estrogen and progesterone receptors in breast cancer. J Clin Oncol 28:2784-2795. doi:10.1200/JCO.2009.25.6529

Hensen EF, Bayley JP (2011) Recent advances in the genetics of SDH-related paraganglioma and pheochromocytoma. Fam Cancer 10:355-363. doi:10.1007/s10689-010-9402-1

Janeway KA, Kim SY, Lodish M, Nose V, Rustin P, Gaal J, Dahia PL, Liegl B, Ball ER, Raygada M, Lai AH, Kelly L, Hornick JL, Pediatric NIH, Wild-Type GC, O'Sullivan M, De Krijger RR, Dinjens WN, Demetri GD, Antonescu CR, Fletcher JA, Helman L, Stratakis CA (2011) Defects in succinate dehydrogenase in gastrointestinal stromal tumors lacking KIT and PDGFRA mutations. Proc Natl Acad Sci U S A 108:314-318. doi:10.1073/pnas.1009199108

Kallinowski F, Schlenger KH, Kloes M, Stohrer M, Vaupel P (1989) Tumor blood flow: the principal modulator of oxidative and glycolytic metabolism, and of the metabolic micromilieu of human tumor xenografts in vivo. Int J Cancer 44:266-272

Kantorovich V, King KS, Pacak K (2010) SDH-related pheochromocytoma and paraganglioma. Best Pract Res Clin Endocrinol Metab 24:415-424. doi:10.1016/j.beem.2010.04.001

King A, Selak MA, Gottlieb E (2006) Succinate dehydrogenase and fumarate hydratase: linking mitochondrial dysfunction and cancer. Oncogene 25:4675-4682. doi:10.1038/sj.onc.1209594

Liu H, Hu YP, Savaraj N, Priebe W, Lampidis TJ (2001) Hypersensitization of tumor cells to glycolytic inhibitors. Biochemistry 40:5542-5547

Miettinen M, Wang ZF, Sarlomo-Rikala M, Osuch C, Rutkowski P, Lasota J (2011) Succinate dehydrogenase-deficient GISTs: a clinicopathologic, immunohistochemical, and molecular genetic study of 66 gastric GISTs with predilection to young age. Am J Surg Pathol 35:1712-1721. doi:10.1097/ PAS.0b013e3182260752

Miettinen M, Killian JK, Wang ZF, Lasota J, Lau C, Jones L, Walker R, Pineda M, Zhu YJ, Kim SY, Helman L, Meltzer P (2013) Immunohistochemical loss of succinate dehydrogenase subunit $\mathrm{A}(\mathrm{SDHA})$ in gastrointestinal stromal tumors (GISTs) signals SDHA germline mutation. Am J Surg Pathol 37:234-240. doi:10.1097/PAS.0b013e3182671178

Moreno-Sanchez R, Rodriguez-Enriquez S, Marin-Hernandez A, Saavedra E (2007) Energy metabolism in tumor cells. FEBS J 274:1393-1418. doi:10.1111/j.17424658.2007.05686.x

Ni Y, Zbuk KM, Sadler T, Patocs A, Lobo G, Edelman E, Platzer P, Orloff MS, Waite KA, Eng C (2008) Germline mutations and variants in the succinate dehydrogenase genes in Cowden and Cowden-like syndromes. Am J Hum Genet 83:261-268. doi:10.1016/j.ajhg.2008.07.011

Pinheiro C, Sousa B, Albergaria A, Paredes J, Dufloth R, Vieira D, Schmitt F, Baltazar F (2011) GLUT1 and CAIX expression profiles in breast cancer correlate with adverse prognostic factors and MCT1 overexpression. Histol Histopathol 26:1279-1286

Ricketts C, Woodward ER, Killick P, Morris MR, Astuti D, Latif F, Maher ER (2008) Germline SDHB mutations and familial renal cell carcinoma. J Natl Cancer Inst 100:1260-1262. doi:10.1093/jnci/djn254

Van Nederveen FH, Gaal J, Favier J, Korpershoek E, Oldenburg RA, De Bruyn EM, Sleddens HF, Derkx P, Riviere J, Dannenberg H, Petri BJ, Komminoth P, Pacak K, Hop WC, Pollard PJ, Mannelli M, Bayley JP, Perren A, Niemann S, Verhofstad AA, De Bruine AP, Maher ER, Tissier F, Meatchi T, Badoual C, Bertherat J, Amar L, Alataki D, Van Marck E, Ferrau F, Francois J, De Herder WW, Peeters MP, Van Linge A, Lenders JW, Gimenez-Roqueplo AP, De Krijger RR, Dinjens WN (2009) An immunohistochemical procedure to detect patients with paraganglioma and phaeochromocytoma with germline SDHB, SDHC, or SDHD gene mutations: a retrospective and prospective analysis. Lancet Oncol 10:764-771. doi:10.1016/S1470-2045(09)70164-0

Warburg O (1956) On the origin of cancer cells. Science 123:309-314

Whitaker-Menezes D, Martinez-Outschoorn UE, Flomenberg N, Birbe RC, Witkiewicz AK, Howell A, Pavlides S, Tsirigos A, Ertel A, Pestell RG, Broda P, Minetti C, Lisanti MP, Sotgia F (2011) Hyperactivation of oxidative mitochondrial metabolism in epithelial cancer cells in situ: visualizing the therapeutic effects of metformin in tumor tissue. Cell Cycle 10:4047-4064. doi:10.4161/cc.10.23.18151

Wolff AC, Hammond ME, Schwartz JN, Hagerty KL, Allred DC, Cote RJ, Dowsett M, Fitzgibbons PL, Hanna WM, Langer A, McShane LM, Paik S, Pegram MD, Perez EA, Press MF, Rhodes A, Sturgeon C, Taube SE, Tubbs R, Vance GH, van de Vijver M, Wheeler TM, Hayes DF, American Society of Clinical O, College of American P (2007) American Society of Clinical Oncology/College of American Pathologists guideline recommendations for human epidermal growth factor receptor 2 testing in breast cancer. J Clin Oncol 25:118-145. doi:10.1200/JCO.2006.09.2775

doi:10.1186/2193-1801-2-299

Cite this article as: Kim et al:: Succinate dehydrogenase expression in breast cancer. SpringerPlus 2013 2:299. 